\title{
Revisiting methodological issues in transcript analysis: Negotiated coding and reliability
}

\author{
D.R. Garrison ${ }^{\mathrm{a}, *}$, M. Cleveland-Innes ${ }^{\mathrm{b}}$, Marguerite Koole ${ }^{\mathrm{b}}$, James Kappelman ${ }^{\mathrm{c}}$ \\ ${ }^{\text {a }}$ Learning Commons, Biological Sciences Building, 530L, University of Calgary, Calgary, Alberta, Canada T2N 1N4 \\ b Athabasca University, Canada \\ ${ }^{\mathrm{c}}$ University of Calgary, Canada \\ Accepted 14 November 2005
}

\begin{abstract}
Transcript analysis is an important methodology to study asynchronous online educational discourse. The purpose of this study is to revisit reliability and validity issues associated with transcript analysis. The goal is to provide researchers with guidance in coding transcripts. For validity reasons, it is suggested that the first step is to select a sound theoretical model and coding scheme. Particular focus is placed on exploring the advantages of the option of a negotiated approach to coding the transcript. It is concluded that researchers need to consider the advantages of negotiation when coders and researchers are not familiar with the coding scheme.
\end{abstract}

(C) 2005 Elsevier Inc. All rights reserved.

Keywords: Transcript analysis; Coding reliability; Coding scheme; Online learning; Community of inquiry

\section{Introduction}

Collaborative asynchronous online communication is becoming a common and valuable technique to engage and sustain discourse in higher education. However, online (text-based) communication is a rigorous and not fully understood educational medium, especially when combined with spontaneous face-to-face verbal dialogue (i.e., blended learning). To date, there is barely beyond an intuitive understanding of the properties and potentiality for online learning. Much work remains in understanding the adoption of communications technology for educational purposes. Due to the complexity of educational discourse, it is imperative to have the techniques and tools to study this educational communication medium.

In this regard, researchers need methodologies to systematically and rigorously study online communication in order to guide educators in the adoption and design of learning in this new environment. The permanent nature of online asynchronous text-based transcripts provides an accessible source to study the complexities of the teaching and learning transaction in this environment. Educators are just beginning a systematic study of how to facilitate an

\footnotetext{
* Corresponding author. Tel.: +1 403282 0730; fax: +1 4032206784.

E-mail addresses: garrison@ucalgary.ca (D.R. Garrison), martic@athabascau.ca (M. Cleveland-Innes), mkoole@athabascau.ca (M. Koole), jkappelman@shaw.ca (J. Kappelman).
} 
effective and efficient online educational experience. One of the methodologies with considerable potential to explore the complexities of online learning is transcript analysis.

Transcript analysis provides a relatively new observational technique to understanding educational discourse in an online text-based environment. The theoretical frameworks and coding schemes/protocols of transcript analysis need to be critically evaluated. Gerbic and Stacey (2005) suggest that it is time that researchers evaluate analytic frameworks in transcript analysis as they "have not been substantively discussed in the literature" (p. 46).

The purpose of this research is to revisit transcript content analysis. In particular, the authors focus on the methodological procedures and coding experiences of a study that used a negotiated approach to transcript analysis. The goal is to provide a guide to researchers in training coders, maintain opportunity for insights, and achieving acceptable reliability measures. The paper begins with a review of the methodological issues (theoretical frameworks and analytical techniques). Reliability results of a recent study are provided next. Finally, a discussion of the advantages and limitations of a negotiated approach to transcript analysis is provided.

\section{Methodological issues}

Reliability and validity issues have their source in the theoretical frameworks, models and coding schemes designed to guide the analysis of transcripts. A sound theoretical framework and model is essential to address validity issues. Coding schemes must be both effective from a reliability perspective and efficient from a resource perspective. A relatively straightforward coding scheme can be of great assistance in the training of coders and the consistent application of the protocol. The categories must be meaningful, indicators must be relatively discernable (i.e., explicit), and message units manageable, if coding is to have reliability. At the same time, a coding scheme that is too simple may limit insights in exploratory research.

A key element in studying online learning and achieving reliable coding is a model and coding scheme that does not introduce added complexity. Coding is a challenging and time consuming task. The model must be parsimonious and yield a coding scheme that has discrete categories and that can be applied effectively and efficiently. One of the challenges is multiple coding, which may suggest problems with the model and coding categories/indicators. While it may be argued that increased complexity may potentially yield a deeper understanding, it can also create inconsistency in the coding process. Theoretical models must be able to be translated into distinct categories and clear indicators that can be understood and consistently interpreted with limited training. Complex models and coding schemes may provide new insights but can have a negative effect on reliability and validity. Parsimony is a great virtue in science and it is also a great advantage for a reliable coding scheme when analyzing transcripts. Researchers have critiqued protocols as not providing discriminant capabilities (Fahy, 2001). Certainly, coding transcripts is a challenge under the best circumstances, but many of the validity and reliability deficiencies can be mitigated with valid models, discrete categories, and clear indicators.

Beyond a sound framework and coding scheme, the unit of analysis is the next problem that needs to be addressed. The goal, as expressed by Rourke, Anderson, Garrison, and Archer (2001), is "to select a unit that multiple coders can identify reliably, and simultaneously, one that exhaustively and exclusively encompasses the sought after construct" (p. 17). Researchers can delineate the unit of analysis at the sentence, paragraph, message, or thematic levels. In determining a unit it is argued that the message unit may be a good compromise; at least in terms of getting a sense of how each of the elements in a community of inquiry interacts to achieve the educational goals. This approach is dependent upon a coding scheme that is not too complex to apply or too detail-oriented. It may be challenging to accurately determine the intent of the message in a single sentence not to mention the amount of coding that would be required to code each sentence. At the same time, using the message as a unit of analysis must be predicated on providing guidelines to participants to keep messages to one or two paragraphs. This is not only good from a pedagogical perspective, it will also increase the chance that the message is coherent and the coding reliable. Regardless, at some point we face the dilemma of breadth vs. depth. Parsimony and reliability are valuable goals; however, this may not contribute to a depth of knowledge (Morse, 1997). Ultimately, it should be the nature of the research question and the purpose of the discourse that determines the model, coding scheme, and unit of analysis. For exploratory research where reliability is less of an issue, it could be argued that the message may be an appropriate unit of analysis as it may reduce decontextualization of the communication. To this point, Cook and Ralston (2003) point out that researchers should pay attention to frameworks and goals and that "the 'context' of conferencing seems increasingly relevant to its analysis" (p. 372). 
Another more practical challenge with transcript analysis is coping with large amounts of data. The issue here is the practical matter of resources and efficiency. One strategy that can be very effective is to sample interaction at specific intervals. This is defensible when there are good theoretical reasons and previous research supports such an approach. This is also appropriate when transcript analysis is only one of several data sources (Gerbic \& Stacey, 2005). If possible, the coding scheme should be used as part of a comprehensive approach utilizing different methodologies and not seen as sufficient in itself. Transcript analysis should be one of several methods and data sources that provide comprehensive interpretation and convergence.

Researchers must select their coding methodology and design their data analysis in accordance with the research question(s). Indeed, multiple methods exist for coding transcripts and choices must be made (Flynn \& Polin, 2003). Some involve a lone researcher coding in isolation according to his or her personal phenomenological point of view. Other methods involve more than one researcher coding identical transcripts for later comparison and reliability analysis. This study describes a negotiated approach. In a negotiated approach, the researchers code the transcripts, and then actively discuss their respective codes with an aim to arrive at a final version in which most, if not all, coded messages have been brought into alignment. It provides a means of hands-on training, coding scheme refinement, and thereby, may increase reliability. The coders gain a new point of reference from which to view the messages as well as the coding scheme. Another advantage of negotiated coding is that it controls for simple errors brought on by inexperience, coder-saturation or misinterpretation. It may also be the approach of choice in exploratory research where new insights are the primary focus.

Another issue to be considered is loss of data. After negotiation, it is difficult to extract the viewpoints of the individual coders. As the coders proceed through successive negotiations, their perspectives become shaped by the negotiation process which irrevocably alters how they approach the coding process. It is unlikely that the coders will be able to return to their original work with the same mindset that they had prior to negotiation. In addition, negotiated coding raises significant questions about what constitutes reliability within the context of transcript analysis. Researchers must grapple with these issues early in the project planning stages.

Despite the challenges and limitations of this methodology, transcript analysis has provided significant insights (Fahy, Crawford, \& Ally, 2001; Garrison \& Anderson, 2003; Gunawardena, Lowe, \& Anderson, 1997; Hara, Bonk, \& Angeli, 1998; Meyer, 2003; Newman, Webb, \& Cochrane, 1995; Vaughan \& Garrison, 2005). Practical and methodological challenges can best be addressed with a sound theoretical framework, parsimonious coding scheme, clear research questions, and a defensible sampling strategy. When these issues are addressed, good reliability measures have been achieved (e.g., Anderson, Rourke, Garrison, \& Archer, 2001; Celetin, unpublished manuscript; McKlin, Harmon, Evans, \& Jones, 2002; Pawan, Paulus, Yalcin, \& Chang, 2003; Rourke et al., 2001; Vaughan, 2004). The promise and demonstrated value of transcript analysis suggest there is every reason to believe that we will see an increased number of studies employing this methodology. Certainly efforts need to be made to address methodological issues, which should start with having research questions that are embedded within an appropriate theoretical framework to facilitate the interpretation of the findings. Following the discussion of the theoretical framework, this paper will present the coding results of a study of interaction in online learning which employed negotiated coding.

\section{Theoretical framework}

The Community of Inquiry (CoI) framework was developed by Garrison, Anderson, and Archer (2000) to guide transcript analysis. The framework had its genesis in the higher education literature, particularly Lipman's (1991) work on communities of inquiry. The framework is composed of three elements - social, cognitive and teaching presence. Each of the three elements was also grounded in the higher education literature. Categories of social presence were generated from an extensive review of the literature on collaborative and mediated communication; the cognitive presence model and categories were grounded in the work of Dewey (1933) (Garrison, Anderson, \& Archer, 2001); and the categories of teaching presence emerged from the literature on teaching roles (Garrison \& Anderson, 2003). During the developmental process improvements were made to improve the discriminatory ability of each of the elements. Examples are provided to assist in understanding the categories and in coding the transcript.

While the CoI framework has not been tested over an extended period of time, it is judged to have good validity based upon its genesis in mature educational literature; the fact that it appears to have some value based upon its adoption to frame online education research; and the fact that there have not been any significant critiques of the 
framework itself. That is not to say the indicators or the categories of the elements are not open to refinement. Much work remains to stabilize the categories and indicators (i.e., coding scheme) across various educational contexts. If used to study questions appropriate to the values and assumptions of the theoretical framework, the coding scheme is applied fairly, and interpretations are comprehensive (confirming and disconfirming data), then the CoI framework should be judged to have validity. The previous are standards for judging the validity of all research (Eisenhart \& Howe, 1992).

Many of the early frameworks focused on either the social community or the learning process. Recently, more interest has been associated with teaching presence. The other core element, cognitive presence, represents a coherent progression or cycle of critical and reflective thinking (i.e., inquiry) as opposed to discrete elements of discourse (e.g., questioning, critiques, explanation, etc.) that may not encourage threaded discussions. The CoI framework provides a comprehensive model that makes possible the consideration of the interaction effects of all the core elements. For example, the effect of teaching presence on cognitive presence can be studied while considering the supporting conditions of social presence. However, if the goal of the research is to study social presence independently, where much of the early online conferencing research was focused, then there is little need for a comprehensive framework and protocol.

As noted, the model can provide the means to achieve internal validity with certain research questions, effective collection and analysis, informed by existing knowledge and the study addresses issues that have value and usefulness. The purpose of the model is exploratory and explanatory in the sense of providing the insights to construct meaningful propositions to be explored in further research. However, external validity is a special challenge in qualitative research; therefore, considerable caution must be exercised as to the limits of generalizability (i.e., how broad is the relevance). However, considering that this research was framed (i.e., constrained) by a credible orienting, conceptual framework, translating findings to similar contexts may be facilitated and warranted.

The CoI model provides a comprehensive perspective on the educational transaction, yet manageable in its application and ease of use. The CoI framework and protocol is a qualitative approach. The coding of the transcripts remains a qualitative analysis, even though frequencies are provided to help gain a quantitative sense of what is occurring. This is appropriate in exploratory research into understanding a new application such as text-based online educational experiences. Transcript analysis framed by this model and protocol is a powerful method to understand text-based educational conferencing and discourse.

\section{Methodological procedures}

At its source, transcript analysis is an exploratory, qualitative methodology. As suggested previously, transcript analysis falls into the qualitative research tradition. The data (text) and analysis (selective coding with constant comparison) are clearly consistent with qualitative analytic practices. However, this is not inductive theory building research. The distinguishing feature of this approach to transcript analysis is that we begin with a credible theoretical framework. As such, the questions emerge from the framework and are deductive in that sense. The framework orients the sampling and analysis. At the same time, this does not preclude inductively derived insights gained through the transcript analysis and, although not reported here, through triangulation with other data sources (e.g., questionnaires and interviews). The analysis may be classified as interpretivist in that during the coding process, we attempt to understand interactions through text analysis (Miles \& Huberman, 1994). Miles and Huberman (1994) state that "qualitative analysis needs to be well documented as a process..." (p. 12). The focus here is to document and elaborate on transcript analysis.

While assigning frequencies to the classifications is an aid in understanding patterns, this does not make it a quantitative, inferential statistical procedure. We are in the early stages of understanding and explaining the complexities of online conferencing and educational discourse. The goal is descriptive, not predictive. This position is supported by Gerbic and Stacey (2005).

A study of student adjustment to online communities of inquiry is the broader context in which the analysis reported here resides. Students participating in this study were enrolled in two graduate programs at Athabasca University. Two core courses normally taken early in each program were purposively selected in order to include the greatest number of novice online learners. Students from 19 distinct course groups over four terms were included in the study. Results reported here are limited to the description of coding decisions and activities. Actual findings of the study will be reported in a subsequent paper. 
All courses were delivered using a combination of print and electronic media, and online conferencing. The online conferencing component provided the opportunity for student engagement and group interaction. Required conference participation was used for assessment in some courses while it remained a voluntary activity in others. Transcript analysis was performed on conferences at the beginning, middle and end of each respective course.

\section{Transcript analysis}

At the beginning of the coding process, the coders received a copy of the Communities of Inquiry (CoI) coding scheme and a set of transcripts from the first classes sampled in the study (The coding scheme is presented in Table 1 and has been described previously under theoretical framework.). At a preliminary meeting with a graduate student who served as a coding trainer, the coders began to code and reconcile a series of online course transcripts. This process helped them to further understand the coding scheme and to familiarize themselves with the coding and negotiation process. Then, the coders independently attempted to code a series of messages from randomly selected transcripts. After the trial documents were coded and reconciled, various issues began to emerge.

The first two issues that arose were in relation to unit of analysis and the coding process itself. Often in online discussions a single message can contain merely a single phrase such as, "Right you are." or, a message may contain any number or combination of sentences, fragments, and paragraphs. In addition, paragraphs and sentences may or may not be well formed. Determining what is or is not a paragraph or another specific unit of communication can become very complex. Based on the exploratory nature of the research, the primary researchers decided that the student's posted message (word, sentence, paragraph, or sequence of paragraphs) should become the unit of analysis. Coding at the message level alleviated the need for the coders to identify units in more subjective ways.

Having determined the most appropriate unit of analysis, the coders had to resolve the complications in the coding process itself. The coding scheme is structured as a hierarchy of presences, categories, and indicators. The three presences, teaching, social, and cognitive, are broken down into categories. In turn, the categories are composed of various indicators. Attempts to code the messages using the more granular indicators became progressively more difficult. The difficulty was compounded by the fact that there was often more than one possible category for each presence and more than one possible indicator for each category. In response, the primary researchers requested that the coders choose only the most salient category for each presence and that they not attempt to code at the indicator level. Aside from reliability challenges, coding at the indicator level was not necessary to answer the research questions of this study. These coding procedure decisions were made early in the project through conversation between the project leaders and the coders. To summarize, the coding procedure was to code for each of the three presences (teaching, social, and cognitive) at the message level. Then, they were to choose only one category for each presence. Indicators were only used to help identify presences and categories of presences.

During the coding process, both coders read through and individually coded each transcript using the coding scheme. The coders applied presence and category codes to each posted message in a transcript. If there was no evidence for a presence, then it received a zero code, CP-Zero or TP-Zero, for example. Under normal conditions, one message received only one category code per presence. At times, when a message contained two equally prominent categories, both categories were indicated because the coders could not agree which category was the most salient. In addition, repeat messages were coded as repeat messages. Messages posted by students who had not given consent to

Table 1

Community of inquiry coding scheme

\begin{tabular}{lll}
\hline Elements & Categories & Indicators (examples only) \\
\hline Cognitive presence & Triggering event & Sense of puzzlement \\
& Exploration & Information exchange \\
& Integration & Connecting ideas \\
Social presence & Resolution & Apply new ideas \\
& Affective & Expressing emotions \\
Teaching presence & Open communication & Risk-free expression \\
& Group cohesion & Encouraging collaboration \\
& Design and organization & Setting curriculum and methods \\
& Facilitating discourse & Sharing personal meaning \\
\hline
\end{tabular}


the study were removed and coded as message removed. In this way, the coders could use the software to search through and count the total number of messages as well as the total number of messages repeated or removed.

After having coded each transcript for all three presences at the category level, the coders met via audio-conference in order to reconcile each message. If they agreed at the presence and category levels, then they immediately moved to the next message. If, at any time, they disagreed about a presence or category, they discussed their reasons for having chosen the codes. If they came to an agreement on the coding of a message, they both changed their codes in accordance. If the coders could not reach agreement, they marked the message as such and moved to the next message. At other times, they marked the message as a question to be directed to the primary researchers. The discussions resulted in either one coder changing his/her code to match the other's code, or both coders agreeing upon a completely different category. The transcript negotiation process was a very slow, arduous task requiring each coder to advocate for his/her codes. Following negotiation of a transcript, the coders conducted frequency counts. Frequencies are counted at the category level. The coders noted the categories they agreed upon as well as the categories they could not agree upon.

In order to better facilitate the transcript coding analysis/reconciliation process, qualitative analysis software was incorporated in the coding protocols. The coders chose software that would maintain both electronic copies of individually coded transcripts as well as reconciled transcripts resulting from the negotiation process. While the software presented certain advantages in terms of report generation, there was a slight learning curve to attain in order for the coders to learn how to use the software, how to set up the codes and coding trees, and how to apply the codes to the messages. The software permitted an easy way to count messages, paragraphs, codes and lines as well as an easy way to search for specific messages that contained specific categorical presence codes. The software also enabled comparison of the coded documents between the two coders. However, the unique feature of the coding software at times hindered the coding comparison between the two coders as the software operated at the character level; that is, one coder could code a document as beginning at character 651, the second coder at character 652 , or another nearby line. While the human mind can ascertain that this is the same message, a computer cannot.

\section{Results of negotiated transcript analysis}

Reliability in the data collection and analysis process is a cornerstone of sound research. Analysis of qualitative, textual data produced by research respondents requires multiple layers of what Giddens (1984) refers to as double hermeneutics: the subjective interpretation of expressed responses relating to concrete experience in the physical world. Raters or coders apply conceptual codes to these expressions, based on their interpretation of that expression. Inter-rater reliability is the measure of the amount of agreement among multiple coders for how they apply codes to text data. Negotiated agreement extends the interpretation of individual raters into a state of intersubjectivity, where raters discuss, present and debate interpretations to determine whether agreement can be reached.

Using the current coding scheme that operationalized key constructs in the community of inquiry, coders in this study were able to achieve agreement on approximately $80 \%$ of all messages. This measure was achieved at the category level for all three presences in the community of inquiry model, suggesting an accurate coding scheme and that the model is a valid representation of the inner dynamics of online conferencing.

Table 2

Negotiated agreement vs. raw agreement

\begin{tabular}{lcccc}
\hline & Transcript 1 & Transcript 2 & Transcript 3 & Transcript 4 \\
\hline Agree & 41 & 14 & 18 & 33 \\
Negotiated agreement & 18 & 33 & 6 & 52 \\
Disagreement & 1 & 4 & 6 & 0 \\
Undecided/questions & 0 & 0 & 0 & 0 \\
Repeat messages & 0 & 1 & 0 & 4 \\
Messages removed & 0 & 0 & 43 & 91 \\
Total messages & 60 & 52 & $7 \%^{\mathrm{a}}$ & $93 \%$ \\
Negotiated agreement & $98 \%$ & $90 \%$ & $41 \%{ }^{\mathrm{a}}$ & $36 \%$ \\
Agreement w/o negotiation & $68 \%$ & $27 \%$ & & \\
\hline
\end{tabular}

\footnotetext{
${ }^{\mathrm{a}}$ This percentage could change as a result of investigating the undecided responses.
} 
Table 2 provides a brief summary of agreement rates at the message level. It is not broken down into actual presences or categories. The columns represent individual transcripts. The rows represent the varying degrees of agreement. Agree refers to the number of messages for which the coders both selected the same categories. Negotiated agreement refers to the number of messages for which the coders disagreed on one or more categories, but were able to reconcile their differences. Disagreement indicates the number of messages for which the coders simply did not agree. Undecided/questions indicates messages with which the coders needed assistance from the primary researchers. Repeat messages were messages that, for some reason or another, were exactly the same as a previous message. They were subtracted from the total messages. Messages removed indicates the number of messages that were removed due to denial of consent. Total messages is the total number of messages in the transcript less repeat postings and messages removed. Negotiated agreement refers to the percentage of agreement achieved after negotiation. Agreement without negotiation refers to the percentage of agreement before reconciliation.

\section{Conclusion}

The purpose of this paper was to review, clarify and offer suggestions to enhance the methodology associated with transcript analysis. The approach described here was to frame the study and analysis within a credible theoretical framework. It is argued that this approach provides increased rigor in coding the transcript. In particular, the paper focused on the challenges of reliably coding transcripts and the advantage that a negotiated approach provides. Practical suggestions were provided to guide those who wish to use this methodology.

Accuracy will not be assured without appropriate structures and procedures in place. Transcript analysis provides a window on actual student behavior in online environments. To ensure clarity of observation in online environments requires the employment of accurate conceptual models and sound operationalization of the constructs within the model. This research demonstrates a number of important factors in such analysis. The coding scheme employed must be of sufficient detail to allow messages to be identified and coded. Inter-rater reliability in cases of rich, sophisticated concepts is likely to require negotiation, a shared identification of meaning.

Use of the current coding scheme requires considerable training and collaboration on the part of coders and researchers engaged in such work. Coders without experience in either educational processes or online environments will require increased exposure and engagement to understand the coding scheme and the responses of research participants. While negotiated coding is particularly useful in exploratory research, another strong argument for negotiated coding is when coders/researchers are not familiar with or experienced in using the coding scheme to analyze transcripts. Even if the coders are experienced in analyzing transcripts, if a new coding scheme is introduced, there may be considerable advantages in a negotiated approach. It may be very beneficial and insightful to explore the different perspectives of the coders. This could add considerably to the development of the framework, refinement of the coding scheme and insights with regard to the research question at hand.

In terms of suggestions for further research, the extent to which negotiated reliability can maintain high reliability at the indicator level could be explored. For example, coders would be assigned to first code at the presence level only, then after a period of time, go back through the document and code at the category level. Finally, after another period of time, coding of same material would occur at the indicator level. Tests of reliability, both 'first-pass' and negotiated reliabilities could be compared across levels. Another way to test the model, the coding scheme, and the strength of negotiated reliability would be to assign the coders to code at the indicator level, and then perform reliabilities without negotiating to obtain raw reliability at the presence, category, and indicator level. Secondly, the coders would then conduct negotiations of the documents to obtain negotiated reliabilities at the presence, category, and indicator levels. These kinds of studies would be very time intensive but would provide a very deep level of analysis for any given document.

Additional research is required on the model and coding scheme. Some segments of the coding scheme used here required more discussion than others. Clarity in the coding scheme and the language used to describe the categories and indicators is essential. Additional work is needed to refine interpretation of the community of inquiry categories and indicators. A parsimonious, well-articulated conceptual model impacts coder interpretation and the ability to code according to an agreed upon conceptual understanding. More research testing this model, the coding scheme, and processes of transcript analysis will bring us closer to an understanding of the intricacies of online interaction and discourse. 
In conclusion, transcript analysis methodologies provide an invaluable technique to understand interaction patterns and the quality of the discourse in online communities of inquiry in higher education (Garrison and Cleveland-Innes, 2005). It is through the use of transcript analysis that educators can investigate beyond what students say they do to reviewing what they actually $d o$. These insights will precipitate further research with other methodologies to identify the principles and practices associated with various educational goals. Ultimately, these results will provide guidance to practitioners in designing online and blended learning experiences in higher education.

\section{References}

Anderson, T., Rourke, L., Garrison, D. R., \& Archer, W. (2001). Assessing teaching presence in a computer conferencing context. Journal of Asynchronous Learning Networks, 5(2) (Retrieved November 11, 2005: http://www.aln.org/alnweb/journal/jaln-vol5issue2v2.htm).

Celetin, P. (unpublished manuscript). Online training: Analysis of interaction and knowldge building patterns among foreign language teachers

Cook, D., \& Ralston, J. (2003). Sharpening the focus: Methodological issues in analyzing on-line conferences. Technology, Pedagogy and Education, 12(3), 361-376.

Dewey, J. (1933). How we think (Rev. ed.). Boston: D.C. Heath.

Eisenhart, M. A., \& Howe, K. R. (1992). Validity in educational research. In M. D. LeCompte, W. L. Millroy, \& J. Preissel (Eds.), The handbook of qualitative research in education (pp. 643-680). San Diego: Academic Press.

Fahy, P. J. (2001). Addressing some common problems in transcript analysis. International Review of research in Open and Distance Learning, $1(2)$.

Fahy, P. J., Crawford, G., \& Ally, M. (2001). Patterns of interaction in a computer conference transcript. International Review of research in Open and Distance Learning, 2(1).

Flynn, T., \& Polin, L. (2003). Making sense of online learning: Frames, rubrics, tools and coding systems for analyzing asynchronous online discourse. Paper presented at AERA, Chicago, April. Retrieved November 11, 2005: http://rs.ed.uiuc.edu/aera/03/communities/Making-Senseof-Online-Lear.pdf

Garrison, D. R., \& Anderson, T. (2003). E-Learning in the 21st century: A framework for research and practice. London: Routledge/Falmer.

Garrison, D. R., Anderson, T., \& Archer, W. (2000). Critical inquiry in a text-based environment: Computer conferencing in higher education. The Internet and Higher Education, 2(2-3), 87-105.

Garrison, D. R., Anderson, T., \& Archer, W. (2001). Critical thinking, cognitive presence and computer conferencing in distance education. American Journal of Distance Education, 15(1), 7-23.

Garrison, D. R., \& Cleveland-Innes, M. (2005). Facilitating cognitive presence in online learning: Interaction is not enough. American Journal of Distance Education, 9(3), 133-148.

Gerbic, P., \& Stacey, E. (2005). A purposive approach to content analysis: Designing analytical frameworks. Internet and Higher Education, 8 , $45-59$.

Giddens, A. (1984). The constitution of society. Berkeley: University of California Press.

Gunawardena, C. N., Lowe, C. A., \& Anderson, T. (1997). Analysis of a global online debate and the development of an interaction analysis model for examining social construction of knowledge in computer conferencing. Journal of Educational Computing Research, 17(4), $397-431$.

Hara, N., Bonk, C.J., \& Angeli, C. (1998). Content Analysis of Online Discussion in an Applied Psychology Course. CRLT Technical Report, 2. Retrieved April 28, 2005 from http://crlt.indiana.edu/publications/journals/techreport.pdf

Lipman, M. (1991). Thinking in education. Canbridge: Cambridge University Press.

McKlin, T., Harmon, S. W., Evans, W., \& Jones, M. G. (2002). Cognitive presence in web-based learning: A content analysis of students' online discussions. ITForum Paper \#60, retrieved October 11, 2005. http://it.coe.uga.edu/itforum/paper60/paper60.htm

Meyer, K. A. (2003). Face-to-face versus threaded discussions: The role of time and higher-order thinking. Journal of Asynchronous Learning Networks, 7(3), 55-65.

Miles, M. B., \& Huberman, A. M. (1994). Qualitative data analysis: An expanded sourcebook. Thousand Oaks, CA: Sage.

Morse, J. M. (1997). "Perfectly healthy but dead": The myth of inter-rater reliability. Qualitative Health Research, 7(4), $445-447$.

Newman, D. R., Webb, B., \& Cochrane, C. (1995). A content analysis method to measure critical thinking in face-to-face and computer supported group learning. Interpersonal Computing and Technology, 3(2), 56-77.

Pawan, F., Paulus, T. M., Yalcin, S., \& Chang, C. (2003). Online learning: Patterns of engagement and interaction among in-service teachers. Language Learning and Technology, 7(3), 119-140.

Rourke, L., Anderson, T., Garrison, D. R., \& Archer, W. (2001). Methodological issues in the content analysis of computer conference transcripts International Journal of Artificial Intelligence in Education, 12(1), 8-22.

Vaughan, N.D. (2004). Investigating how a blended learning approach can support an inquiry process within a faculty learning community. Doctoral dissertation, University of Calgary.

Vaughan, N., \& Garrison, D. R. (2005). Creating cognitive presence in a blended faculty development community. Internet and Higher Education, $8,1-12$. 